\title{
Determining the Necessary Requirements for Developing a Business-to-Business Application: A Consultancy-Based Analysis
}

\author{
A. I. Haruna
}

\section{ABSTRACT}

Digital transformation, one of the by-products of technological advancement, has become a powerful driving force for success in the corporate world. The reasons are many and varied: the use of technology helps expedite business processes, manage employees remotely with ease while ensuring smooth automation of various operations. However, in this age of unprecedented volatility in the market, all business leaders need to think differently about their organization's strategic direction and prepare their businesses for the disruptive competition that lies ahead. It, therefore, makes business sense that Company $X$ is contemplating developing a mobile Business-to-Business (B2B) application to help boost businesscustomer interactions. That, in turn, would ensure profitable business growth that maximizes shareholder value and remove the core barriers and restrictions that hinder business growth. As a Small and Medium-sized Enterprise (SME) in the food industry that supplies food products to catering companies, Company $\mathrm{X}$ has realized that implementing a welldeveloped and customized B2B mobile app in the daily transactions of the company would be one of the surest ways to increase visibility and maximize sales. The ensuing pages and paragraphs would present a report bid proposal for Company $X$, which requires a scoping study to determine the needed requirements for developing and implementing the Businessto-Business (B2B) mobile application. For the app to successfully address the needs of the business, and be widely accepted by customers, several factors must be taken into consideration before, during, and after the development of the app. Thus, this report would focus on addressing those existential requirements that the client needs to analyze carefully before allotting any resources - both financial and human - to the project. The purpose is to make available to Company $\mathrm{X}$ a strategic decision-making tool that would aid in pre-investment brainstorming and preparations. The bid report adopts industry-approved methodologies and techniques to examine, scientifically, the most suitable structural, resource, and functional requirements required for the development and effectuation of the mobile Business-to-Business app project. Given that the timeline for the completion of the study is six (6) months, this report would rely on the Gantt chart to outline the sequence of activities and to determine when, where, and how each activity would be performed. This is meant to ensure effective and efficient completion of the report.

Keywords: Business-to-Business, Company X, Consultant, Gantt Chart, Medium-Sized Enterprise, Mobile Application
Published Online: January 21, 2022

ISSN: $2736-5522$

DOI: $10.24018 /$ ejsocial.2022.2.1.196

\section{A. I. Haruna}

PhD Candidate: International Relations, Social Sciences, University of Ankara, Turkey.

(e-mail: harunaimm ${ }^{\circledR}$ yahoo.com)

\section{INTRODUCTION}

The development of information and communication technology (ICT) across the globe has, in a very impressive and drastic manner, overhauled and accelerated the way we communicate and do business (Okello-Obura \& Ssekitto, 2015; World Economic Forum, 2003). According to DATAREPORTAL (2021), as of July 2021, while 4.80 billion (60.9\%) people worldwide use the internet, 5.27 billion (66.9\% of the world population) are active mobile phone users with 4.48 billion $(56.8 \%)$ actively engaged in social media. 3.8 billion out of 5.27 billion mobile phone users own smartphones. Kapil Mehta (2017) postulates that on average, people spend 2 hours daily on their mobile phones as compared to a little over an hour they spend on family and friends. According to the Mobile GSMA (2018), by 2025, 71\% of the global population will be unique mobile phone subscribers. Smartphones have the propensity to create and maintain mobilefriendly websites for business growth. In effect, these internet-enabled devices such as tablets, smartphones, and laptops, among others, have driven business growth and changed the way consumers behave in recent 
years (Aldhaban, 2012; Dahlstrom et al., 2015; Daniel, 2016; Samsung for Business, 2021).

Therefore, mobile phones have now become the most convenient point of convergence for corporate organizations, such as the hospitality industry, the aviation industry, financial institutions, as well as those in the e-commerce sector. These corporate organizations and other business entities now capitalize on the smartphone technology and the accompanying business-to-business applications to increase their visibility among their audiences and boost their sales (Chang, 2016; Gill et al., 2017; Kuhr, 2017). In other words, because many mobile phone users now spend more of their time on these mobile applications, "firms are increasingly offering engagement initiatives to facilitate firm-customer interactions or interactions among customers, with the primary goal of fostering emotional and psychological bonds between customers and the firm" (Gill et al., 2017, p. 45). Businesses that do not take advantage of the technological innovations to reach out to their customers are more likely to lose valuable customers to competitors.

Company X, the client under discussion, is a Small and Medium-sized Enterprise (SME) within the food industry, providing food products to companies engaged in catering services. Being conversant with the influence of electronic devices on the corporate world (Gupta et al., 2013), the company is seeking the services of an advisor to do an evaluation study to determine the needed necessities for creating a mobile B2B app to increase the company's visibility and boost its Business-to-Customer interactions. The app, among other things, will also provide value to the company's customers; enhance the company's brand recognition, and produce a direct marketing platform with clients to promote the products of the company (Bharadwaj et al., 2013; Dan, 2017; Rose, 2017). Thus, this bid report is aimed at offering the needed consultancy and/or expert advice to the client using industry-based approved methodologies to sketch out all relevant requirements, which are of significance for ensuring the success of the project.

\section{A. Study Objectives}

As we have already discussed, Company $\mathrm{X}$ is an SME providing food products to the catering trade. Therefore, to help ensure a competitive level of service, the company is considering the suitability of creating a mobile app that would ensure a consistent B2C interaction. The discussion in this report is therefore geared towards identifying the requirements for a successful B2B mobile application development for Company X. In keeping with these methods of this report, the following objectives will be pursued.

Objective 1: Set up the functional necessities for developing a mobile B2B app for organizations rendering catering services. Several analyses, including security requirements analysis, market and stakeholder analysis, and software benchmarking analysis are done here.

Objective 2: Identify the structural requirements for the development, deployment of the mobile B2B of the app in keeping with the capabilities, customers' needs, and organizational structure of Company X. To this end, personnel analysis, analysis of system environment as well as identification and analysis of risk will be performed.

Objective 3: Identify and establish the resources needed by Company $\mathrm{X}$ to build, install, and operate the mobile B2B app. A Life-Cycle Cost Analysis and a project time planning analysis will be performed to ensure the effective realization of this goal.

\section{B. Methodology}

It is argued that while the success or otherwise of most software is largely influenced by the caliber of the necessities obtained, the quality of those necessities is also highly dependent on the methodologies applied to assemble these requirements (Fahad, 2016). Fahad (2016) postulates that one of the critical success factors for the expansion or growth of the Business-to-Business environment is the accessibility of data - both internally and from partner agencies. Given that both requirements engineering and requirements elicitation are employed in the development of software, this report will employ case studies that are industry-oriented to better comprehend the diverse project requirements. Since requirements elicitation is considered the most important step in software design, the study focuses on the requirements elicitation approach, in which different users, customers, and stakeholders of B2B mobile applications are approached, as recommended by industry experts (Dolly \& Khanum, 2016; Gunda, 2008; Lent, 2018).

While the research will do a critical examination, and justify the techniques employed, an organizationwide evaluation tour is believed to offer meaningful data for the development of the app and its content. It is hoped that by striving to correctly define these necessities, the quality of the requirements would increase, and therefore the quality of the final software application. Thus, to achieve the highest quality of research, the proposed review will focus on pointing out the secondary concern and breadth behind the selection of techniques used. In this sense, the involvement of specific techniques will explain why the methodology presented is the most valuable to Company $\mathrm{X}$. The closing comment will abbreviate the key details of the proposal to give a formal conclusion and direction to the proposal. 


\section{SUPPORTING EVIDENCE}

In this section of the report, an attempt is made to situate the discussion within the context of the client's project requirements, and the procedures that correspond to the chosen methodology for the report as discussed above. Different methodological tools such as brainstorming, prototyping, interviews, group discussions, and market analysis, among others, are used to collate data for the analysis. To this end, the need for unending communication and the sharing of relevant information between the consultant and the client throughout the requirements elicitation process cannot be overemphasized. This is to ensure that changes and afterthought requirements and plans can quickly be evaluated on time to integrate them into the initial plans without an undue delay. The choice of this method is anchored on the experience of Rafiq et al. (2017), who said, "requirements elicitation is an ongoing process with numerous changes occurring throughout and after the requirements identification process." Therefore, the need to stay in constant contact with business leaders and/or administrators cannot be overemphasized.

\section{A. Objective 1: Functional Requirements}

As I have already indicated above, in today's dynamic and complex corporate world where technology consistently advances, the use of mobile devices has taken on a new dimension. With mobile devices being used on the go, every business, either small or medium-sized businesses, or even larger ones, needs to create a mobile application at some point so that customers can access their services anytime from anywhere. Oinas-Kukkonen et al. (2013) identify two types of mobile applications: entertainment-focused and highly goal-oriented applications. The difference lies in the functionalities and characteristics or features, which can change depending on the operational functions of the application. The challenge in today's market is to develop an app that adds value to the business. It is important to indicate that some other experts identify more than two types of mobile applications. For instance, Lionel Valdellon (2020) argues that we can differentiate between three basic types of mobile applications if we categorize them based on coding technology.

In the end, the type of mobile app one chooses to build "is not a one-and-done decision." It is always possible to switch to another type down the road for better results, depending on the needs of the business and that of the customers. However, since Business-to-Business mobile apps face acceptance challenges among a sizable number of customers, perhaps due to a lack of orientation and relevance to the user, for the application to be successful in the market, it is advisable to study how the app will work and how useful it will be for the user. This report, which among other things, analyses the functional requirements of the B2B app, seeks to advise the client on best practices regarding the functional operations of the application, its usability, and security that comply with the business-to-customer relationships and purchasing strategies.

\section{Stakeholder and Market Analysis}

Irrespective of the target audience and the objectives of the mobile application, it is always advisable to do market and stakeholder analysis to guarantee an informed decision that would ensure the success of the B2B application. For instance, a thorough understanding of customer behaviour and the characteristics of the target market is indispensable when designing the B2B app and the corresponding functionalities that would fit the characteristics of the target market. In other words, keeping abreast of the needs of the market and the unpredictable behavior of the customer will help the designers of the application develop an app that is unique to the target market and thus serves the customer better.

To this end, conducting market research will help in understanding the customers' preferences or expectations. This is very important in determining how to integrate any business application. For example, it is argued that if B2B mobile application developers are unable to appreciate the needs of the app's endusers, this will reflect on the app's features and functions, lowering the app's market acceptance rate ( Robertson, 2005). Therefore, to be able to apply user-centered approaches to the design of the application, it is advisable to analyse the market thoroughly and get an appreciable understanding of the target customers. The market analysis will give the designer the practical details of the available hardware and operating systems that can support the smooth running of the application. Besides, it is not in dispute that the type of operating system used in designing an application affects the market price and usability of the app. The market analysis is also crucial in describing the stakeholders of the project, and in revealing Company X's comparative advantages over its competitors in the market. This will help the company launch a successful entry into the market. Because of this, this bid report will also, among other things, identify the potential users of the app to determine the best workable platform for the application, either on a new device or on existing mobile devices. The required features for an easy-to-use app, the transaction rate that would be permitted on the app and all aspects of the app to which customers are allowed access or restricted have to be examined.

As part of the market analysis, there is the need for a special focus on stakeholder analysis. Analysing the characteristics and concerns of stakeholders is a critical process of requirements elicitation that acknowledges the fact that business entities operate in a complex social context where different actors with 
varied concerns and visions can affect (either positively or negatively) the prospects of the business (Eversole, 2018). On one hand, stakeholders in the general gumption can be identified as individuals or corporate entities who have stakes in an organization and can influence or be influenced by that organization. On the other hand, stakeholders in software development represent groups or individuals and organizations that influence or are influenced by the creation of application systems (Chibesakunda, 2015; Ryan, 2014; Sharp et al., 1999). This handy and practical tool makes it possible for us to identify who the potential stakeholders would be, define their expectations and deal with their specific needs. At the end of the day, the yet-to-be-developed software can be tailored towards working with the stakeholders effectively.

Arguably, stakeholder analysis should begin with exploring the identity of all potential stakeholders, their unique features, and the evaluation of their impact. In one sense, this helps in developing a targeted strategy with the stakeholders in mind. In another sense, identifying and analysing the stakeholders provide a detailed understanding of an organization's outcomes and the social context within which it operates. In this regard, Eversole (2018) admonishes that clients with in-depth expertise in internal and external procedures need to be involved. To ensure the full participation of the stakeholders and understand their specific needs from the outset, it is crucial to have a one-on-one discussion with them, or better still, they could be engaged in structured or semi-structured discussions. In fairness, not all the potential stakeholders will have equal investments in the project under consideration. Thus, it makes business sense to come up with a stakeholder matrix that prioritizes their needs, contributions, expectations, and/or goals.

\section{Testing Software Benchmark}

Although various business-to-business mobile applications in the market are designed for smart devices and are simpatico to multiple operating systems, those apps offer distinct user experiences that are associated with customer satisfaction or gratification (Pizzutillo, 2015). Just like all other ubiquitous electronic devices, mobile phones suffer from a scarcity of resources. Hence, corporate organizations need to perform software benchmarks when developing apps for their businesses and before entering the ebusiness market (Rwassizadeh, 2017).

Generally, benchmarking can be seen as the comparison of services, products, and processes between departments performing similar activities within the same organization, or between competing companies in the same industry, and between companies with similar processes in different industries (Robertson, 2017; Fuller \& Robinson, 2015). The ultimate goal of benchmarking is to uncover why and how an organization achieves higher levels of performance and to identify the methods and processes that lead to this performance (Michael, 2017; Strang, 2010). In the field of software development, benchmarking could be seen as the process of comparing available data from a multiplicity of sources to ascertain the performance rate of teams (Pang et al., 2006). In the case of this report, the process is analysing the possibility of existing practices in the market, which could be compatible with, or match the stakeholders' objectives of Company X. Comparing a company's performance and/or operations with that of industry best practices helps in identifying critical information to improve the company's operations management and thus, improve performance. In this case, benchmarking is nothing less than a competitive analysis. The analysis does not only explore best practices but also consults with software companies to discover the most specific characteristics of participation. In the end, this will help define the client's functional requirements to a certain extent that permits him (or her) to gain an edge on the competition over other competitors in the industry in terms of both technology and quality of service.

In analysing software companies and industry rivals (or competitors), the process should begin with need-based analysis, domain ranking or classification, and the gathering of qualitative data from industrycertified databases. After the collection of data, there is a need to refine and normalize the information so that it can be fit for purpose. The analysis should compare the identified objectives of the stakeholders with developed standards or benchmarks to determine the ultimate requirements that the B2B mobile application has to meet to gain a competitive advantage in the marketplace. The benchmark report ends with recommendations on how to improve performance (Kasozi, 2016; Vorhies \& Morgan, 2005).

\section{Security Requirements Analysis}

As I have previously indicated, over half the world's population has access to the internet and one form of an electronic device or the other. However, the global systems and infrastructure that govern access to these 'by-products' of technology are on a knife-edge. The world needs a new bold approach to secure the future of unrestricted access to these products of technology. Security is even more essential when it comes to mobile application development because these apps have become integral parts of our corporate transactions. Indeed, it is argued that the security requirements for corporate apps and that of mobile applications are almost the same, as people outside a company often carry confidential information with them. Not only are these cell phones often unsafe, but users are also exchanging information on insecure networks on the go, which requires figuring out the requirements to find out how the data can be protected. In other words, like desktop computers, mobile devices (tablets, smartphones, notebooks, and laptops) are widely used to access and store both institutional and personal data and information. However, due to their 
portability, mobile devices are more prone to lose and theft than desktop systems. Thus, applying appropriate safety measures to these devices to mitigate the risk of data loss and information exposure due to theft or loss is of paramount importance to app development. Simply put, security should be the heartbeat of any mobile application.

It is extremely difficult, according to Pizzutilo (2015), to ensure the safety of data on devices that are not associated with any data protection organization. Perhaps, the most effective and secure method of safeguarding mobile data is protecting the mobile device itself. It is also advisable to limit the visibility of personal data by enabling security features on smartphones while ensuring the availability of the company's data to prevent any data loss. This bid report will thus advocate strongly for significant security requirements for data allocation offline, the authentication and protection of file systems, hardware and network options, and resource authorization, among others. Based on the recommendations of experts in software development, the Common Vulnerability Scoring System (CVSS) should be used by the consultant to identify the key features of risks and vulnerabilities associated with mobile applications, determine their severity of character, prioritize the components and make proposals for vulnerability management processes (Prasad \& Kishore, 2013; Smriti \& Maya, 2011).

\section{B. Objective 2: Structural Requirements}

This section intends to demonstrate how this bid report examines the structural prerequisites required by the client to be able to develop, run and maintain a successful Business-to-Business mobile app that would meet the expectations of the complex corporate world.

\section{System Environment Analysis}

Several studies show that B2B mobile apps have seen exponential growth in the last decade. With this comes a lot of competition, where each of the apps is competing for space and recognition among customers. The saturation of these applications in the market makes it extremely difficult for corporate organizations to integrate new specific application functions into the existing interfaces and IT systems so that customers will be able to discover and download these applications, which will provide them with the best user experience. This problem, coupled with the fact that software benchmarking shows the functional requirements that correspond to stakeholders' priorities and/or objectives, and re-examines their priorities in the stakeholder matrix; the system environment analysis ought to examine the ease of developing and implementing these necessities in Company X's IT system environment. The integration of changes into this system environment of interactive systems that are part of the operating company and that involve other entities creates restrictions that must be checked to see if the customer can manage them. Thus, to be able to decide what specific necessities are needed for the new system, the consultant will have to work with various stakeholders.

There is also the need for the study to examine the needs, compare the interfaces and the systems before and after the development of the new application. A graph or chart should be created to facilitate the understanding of the affected interface tools and modules required for data exchange and functional use of the app. The study also needs to disclose the existing support processes and IT services that need to be considered during the development and implementation of the B2B mobile app. To do this, the process would have to include interviewing the experts in IT systems and/or application owners to obtain up-todate applicable data about the system and with external suppliers, in case external stakeholders administer the IT processes.

\section{Personnel Analysis}

Based on the identified functional requirements that have to be integrated and implemented and their interaction with the system environment, the personnel analysis seeks to examine whether Company X has the human resources necessary for the implementation. The personnel analysis is necessary because the development and implementation of a mobile B2B app can be successful only if the client has the right team' with the needed acquirements.

There are many ways of enhancing the expertise of employees, for instance, by subjecting staff members to proper training. However, that could prove a bit cumbersome for this new software project. Thus, comparing the roles and tasks in the client's company would help in understanding the specific requirements and shortfalls of the staff. Hence, the need for personnel needs analysis (OECD, 2004). To this end, the first step in this project should be the implementation of role planning, where roles are outlined based on the defined functional necessities and the outcome of the analysis. Nevertheless, the functions of the project also have to be integrated into the structural hierarchy of the client, taking into consideration the administrative and technical tasks. As the project entails new roles and may enforce add-on tasks, the institutional capabilities of the client need some consideration based on internally collected information such as organizational charts, personnel databases, and job descriptions, among others. Consequently, an interview with the chief executive officer or any influential member would be very instructive. 


\section{Identification of Risk and Analysis}

Dickson (1989, p. 2) sees risk as "[T] he identification, analysis and economic control of those risks which can threaten the assets or earning capacity of an enterprise." The effects of the risk could be either positive or negative. The future is unknown, and for project management, this means that decisions are made under uncertainty at all stages of the project. To support this decision-making process, a series of risk and uncertainty analyses should be carried out during the project period (Hood \& Nawaz, 2004).

Admittedly, all projects come with specific risks. Thus, it behooves the project manager to identify and deal with these risks throughout the project. While eliminating these risks may be impossible, a careful approach to managing project risks is among the most important steps in the planning and execution of any project (Sandy et al., 2005). As Company X strives for sustainable results and benefits from the app in the future, it is important to identify the potential risks that could affect the success of the project. This would help the client determine the level of risk that is acceptable to the company.

The risk identification process should be based on the knowledge of other projects of other clients. The checklist of potential project risks could be the starting point. A matrix should be provided with an ordinal scale (high, medium, low), which indicates the likelihood of risks and their magnitude, as well as how to address those expected risks (Boehm, 1991). This gives the client an inkling of whether the risk would be manageable or not.

\section{Objective 3: Resource Requirements}

After reviewing the diverse required structural necessities for implementing the project in a manner, that fulfils the previously defined stakeholders' objectives, the project advisor would then investigate the necessary resources for the effectuation of this mobile business-to-business app. The resources might include tangible facilities, measurable equipment, or factors required for the implementation of the project (Hartney, 2017). In software development, devices can indicate tools in various updated versions, hardware, etc. that the organization can either rent or buy (Jennifer, 2017). Irrespective of the nature of the resources, they ought to be accessible throughout the duration of the project until they are exhausted. The study, in addition, looks at the life cycle of the project and the necessary funding that the client needs to do the implementation.

\section{Time Planning of Project}

The B2B app development, as with most business projects, is a client-based project. Among the long familiar temporal requirements in software engineering is project scheduling, which allows for the estimation of the time that Company $\mathrm{X}$ will need for the execution of the project. Because any project is deemed to have succeeded when the demands of everyone involved are fulfilled; and given the complexity of the industry, which harbours many uncertainties, time planning is the most important step in software engineering.

Time planning in the software business begins with breaking down network-based activities to estimate the amount of time necessary and build the timetable. This stage involves an extensive understanding of software development, which necessitates the use of professional software engineers. However, prior case studies may also serve as a guide, which is important for predicting the necessary timing for the operations of all networks. As advised by the Project Management Institute (2013), after figuring out the networkbased operations, the subsequent step is to organize them logically and in keeping with their dependencies. Al-Naeem et al. (2005) indicated that the estimation of the duration of every activity is vital for determining the overall length of the job and is important in constructing a network diagram, which leads to a complete Gantt chart.

\section{Life Cycle Cost Analysis}

The Life Cycle Cost Analysis (LCCA) of the project is among the suggested procedures for determining the economic worth of a project by assessing initial and future expenditures such as project maintenance, rehabilitation, reconstruction, resurfacing and restoration. This method assists organizations in making value-for-money investment decisions over the course of a business venture. Even though costs within the software enterprise are not constantly apparent without thorough analysis, the Life Cycle Cost Analysis appears to be the right method for determining the overall monetary requirement for the mobile B2B app as it shows not only the initial costs but also the follow-up costs over the lifespan of the project (Douglass, 2016). Fuller (2016) postulates that life-cycle cost analysis (LCCA) is a method for calculating the entire cost of a project from project design through project completion. It considers labour, material, and other expenses, which can be categorised into five classifications: contingent, direct, indirect, intangible, and external costs. While the time plan approach separates tasks into phases, the LCCA technique makes use of the findings to decide the complete costs of the project, which can be derived via the addition of the expenses of individual activities. The cost of the individual activities is evaluated using existing market pricing for hourly wages or equivalent tools (Najadat et al., 2012). 


\section{Study GantT Chart Method}

As previously stated, the study will employ several techniques to carry out interdependent tasks. Because each assignment has a certain implementation time and length and must be completed in a specific order owing to their interdependence, utilizing a Gantt chart study, as indicated by Chinampton (2011), will be a good technique to show the progress of the project. The Gantt chart, pioneered by Henry L. Gantt (18611919), is a "simple representation of a project from the view of the time taken for each activity and the resources required at any point in time." The tool depicts all activities of the project; and timetable, which shows "the duration of individual activities and their interdependence, the possibility of activity overlap, and the start and finish dates of the overall project."

Therefore, the study focuses on the fundamental project's activities within a 6-month or a 24-week duration. The Gantt chart would guarantee the execution of all planned activities and ensure the feasibility of contingency plans in case the schedule encounters impediments. The study begins with an initial meeting to start the project. This initial meeting is meant to introduce the project to all the significant stakeholders, introduce all participating and accountable individuals, and offer project specifics (Aston, 2016). The study is broken down into separate sections that correspond to different objectives aimed at satisfying the requirements of the client.

Below is the Gantt chart showing all the activities in two dimensions. While the required sequential activities to accomplish the objectives and successfully and efficiently execute the project are displayed in the vertical axis, the horizontal axis indicates the duration for each activity and their respective implementation time. The significance of this, among other things, is to help the stakeholders and the client, in particular, to understand the key steps of the project and concur on the anticipations that Company $\mathrm{X}$ expects from the project consultant during the progress and feedback reports' meetings.

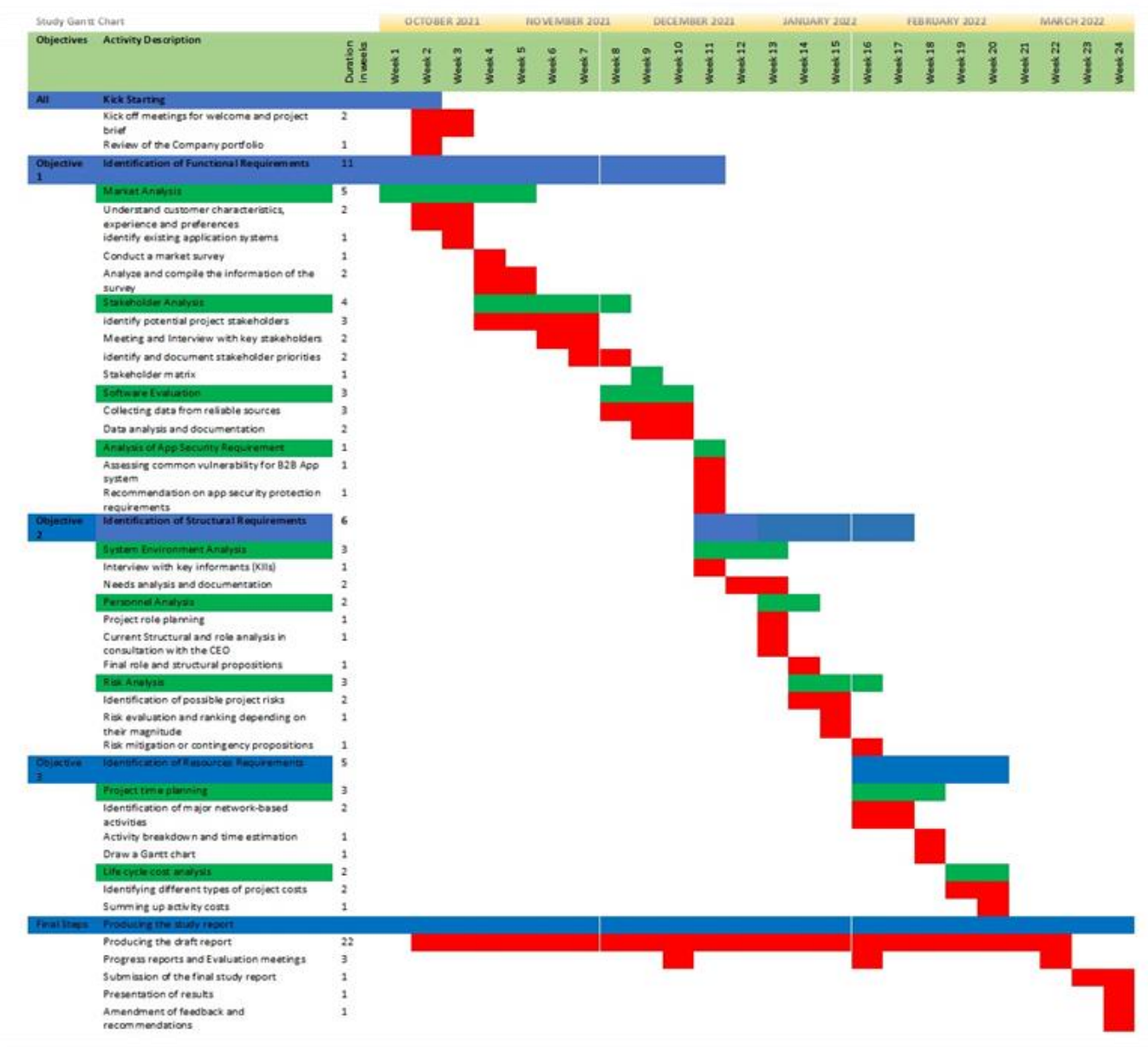

Fig. 1. Gantt Chart (Source: Researcher's Own Figure)

\section{CONTRIBUtions}

This part combines sector research, industry evidence, and past case studies, to demonstrate how the 
preferred methodologies add value to the stated objectives of the study and ensure the client's satisfaction of overall requirements.

\section{A. Objective 1: Functional Requirements}

The functional requirements, as Veronica et al. (2014) have indicated, depict the services of the B2B application system. Based on the scope of the project, the study focuses on the requirements of the company, stakeholders, and users for which the following methods of obtaining requirements are advocated by previous researchers in the industry.

Stakeholder and Market Analysis

To add more value to the performance of the project and guarantee the success of the project, Rolstadås et al. (2015) believe that market analysis is an essential decision-making tool in management, which can assist managers to accomplish their organizational set goals without much difficulty.

Applications in the food industry do not seem to yield profitable returns for investors because app developers ignore certain market prospects. As Haugestad (2015) recommends, conducting a market analysis before the development of a B2B application is a fundamental value-based approach that would help in facilitating smooth interactions between salespeople and prospects and provide supply-sourcing opportunities. In B2B sales, on the other hand, the decision-making process often involves multiple stakeholders with diverse capabilities and interests (Apollo, 2018). Given this, the stakeholder analysis is necessary for Company X to discover those who are most likely to influence the mobile B2B app project, and deal with their varied priorities and needs. Thence, the study would reach out to the previously identified potential stakeholders to solicit information on the design and functionality of the application. This would ensure that stakeholders' interests are properly aligned with the objectives of the project, which would in turn guarantee the success of the project.

\section{Software Evaluation}

Among the several vital steps in the development of the mobile business-to-business app is application benchmarking. The benchmarking process compares the overall performance of an application with its counterparts in the industry and allows for an objective assessment of the app (Pizzutillo, 2015).

While acknowledging the fact that adopting functional features from other applications could have adverse effects on software development, it is without a doubt that some functionalities are markedly unique and suitable for particular industries. Thus, it would be proper to identify and adopt those functional features that have the potency to improve the performance of the business. After all, improving on existing technologies is as good as inventing new ones. In respect of this, the benchmarking process would help Company X enhance its IT systems and be able to discover some missing or unused features and functions. Conventional wisdom within the corporate industry indicates that benchmarking mobile applications can unveil hidden functional requirements that would not have been discovered by the basic processes of the stakeholder or market analysis (Barnshaw, 2016; Duffie et al., 2017). For instance, Q/P Management Group Consultants (2018) have conducted several benchmarking studies to evaluate software development companies and compare them with industry best practices. Many large corporations, government agencies and outsourcers also use project benchmarks and applications. It is very important to compare similar applications in terms of resource usage (Rawassizadeh et al., 2011). According to research and benchmarks from some popular messaging applications such as Viber, Facebook Massager, WhatsApp, Telegram, and Skype, reveals that Telegram users consume more data than the other messaging apps (Daniel, 2017).

\section{Security Requirements Analysis}

The essence of security in mobile application development cannot be overemphasized. This is so because mobile applications' security requirements are not as those of other applications in software engineering (Bedell, 2013). With more sensitive business and personal information leaking through electronic devices especially, mobile phones, it is advisable to critically look at the security requirements prior to the development of a mobile application to be able to detect, analyze and manage potential threats or attacks from various actors (Jeon et al., 2012). The security requirements are, thus, part of the essential steps towards the enforcement of organization-defined restrictions to ensure data protection.

\section{B. Objective 2: Structural Requirements}

\section{System Environmental Analysis}

It is highly advised to conduct the "system environmental analysis" because it can appraise the factors that would alert Company X about potential obstructions associated with new systems, and to consider adopting emerging technologies as well. Concerning this, we are examining long-term and large-scale technology developments. This approach helps us, in particular, to draw conclusions about employees' qualifications and skills requirements, and the potential IT risks, as different IT environments require specific skills to reduce the risks that are associated with failures. Since organizational politics, poor human interactions, technical issues associated with IT, and jejune adoption of software engineering practices have, 
most often, been the cause of many of the failures in software projects in Nigeria and elsewhere in Europe, the recommendation is that they should be deflected through a detailed analysis of Company X IT infrastructure (Walid, 2017).

\section{Personnel Analysis}

The nature of a client's project directs or determines the trajectory of the personnel analysis. In the case of our client, the main components of the project include developing, implementing, and maintaining a business-to-business mobile app. Achieving these steps throughout the lifespan of the project would require a wide range of procedures, services, and the required tools to guarantee that the right functions are performed by those with the right expertise. Thus, the services of highly qualified personnel such as administrators, designers, developers, among others, would be hired to perform the daily tasks of the project. In this context, we would advise Company $\mathrm{X}$ to elicit the scoping requirements based on applicable case studies to illustrate the needs of its personnel. Indeed, personnel for software development is not just developers. They are "tight knots of various skills crucial for a given phase of a project, possessed by various specialists." Therefore, we cannot hope for the success of any app development project without the appropriate deployment of the most qualified personnel (Krikor, 2010). Hence, aside from showing the need for qualified personnel, the personnel analysis also aids our client in the decision to either restructure existing internal employees or outsource skilled labour from external sources.

\section{Identification of Risk and Analysis}

While Henry (2004) sees project risk as "an event, development, or state in a software project that causes damage, loss, or delay", Schwalbe (2004) thinks that project risks are "...problems that might occur on the project and how they might impede project success." Given that our client is entering a new area of IT, where risk is not unexpectedly high because of the varied stakeholders involved and the threats that come with an unfamiliar environment, it is beneficial to identify and analyse the potential risks to be able to manage them when they come (Ahmed, 2017). IT researchers and professionals recommend that project managers perform risk identification and analysis before starting an IT project (Gotterbarn \& Rogerson, 2005). This helps in understanding the characteristics of potential risk; and adopting the appropriate measures to avert them (Verdon, 2004). Additionally, risk analysis should ensure optimistic estimates on cost and time, guarantee adequate records on stakeholders' inputs, and provide prompt reviews on customers' feedbacks (Haughey, 2015).

\section{Objective 3: Resource Requirements}

As revealed by Adrienne Watt (2014), three non-functional development constraints exist in software design. These are time, resources, and quality. It is very significant, practically, to know the magnitude of the requirements during particular software development. This would be useful in evaluating the change magnitude in the project requirements and estimating the financial commitment needed for the development and/or maintenance tasks.

\section{Project Time Planning}

There is a corporate adage that "failure to plan is planning to fail." Time scheduling in project management is crucial in deciding projects' requirements and fixing deadlines for a successful delivery (Chitkara, 2014). In other words, "if time is managed well, other factors are much easier to manage, and projects will be more effective and efficient" (Haugan, 2001: 1). This makes project planning an indispensable component in the successful delivery of any project. If the project time is wrongly estimated, it can result in late delivery, which would negatively affect the cost of the project (Bottlik, 2017; Liz, 2018; Tira, 2018). Incorrect scheduling can result in inadequate requirements, which, in turn, could heighten time constraints for projects. It is, thus, advisable for the client to do a conscious project planning analysis to avoid poor scheduling and all the risks associated with time losses.

\section{Life Cycle Cost Analysis}

Cost, according to Passenheim (2009), constitutes one of the key requirements of project implementation. The Corporate Finance Institute (CFI) describes Life Cycle Cost Analysis (LCCA) as "an approach used to assess the total cost of owning a facility or running a project." It "considers all the costs associated with obtaining, owning, and disposing of an investment." The process helps project managers to come out with the cost that will ensure value-for-money without compromising quality. This approach is also applicable to the B2B mobile app development, serving as a strategic decision-making tool for the project designers. Tripathi and Rai (2016) highlight how beneficial costs estimations are in software design in determining the success or failure of project execution or contract negotiations. This will invariably assist the client to be able to galvanize the needed resources to ensure the success of the project with minimal overall cost. 


\section{CONCLUSION}

This bid report employs a holistic approach to identify and analyse the necessities of the B2B mobile app project, which the client requires to make a cogent investment decision. Given that the technique adopted involves diverse knowledge-based and proven methodologies, every analysis has been tailored to meet the specific needs of the client to enable Company $\mathrm{X}$ to obtain suitable requirements to boost its business performance. Research in the field of software development is indispensable in demonstrating the accuracy of the chosen methodology to determine the needs of the mobile Business-to-Business app for our client.

Although the Gantt chart indicates perpetual client engagement throughout the project, the consultant seizes every opportunity to stay in touch with the client to be able to address any changes in the requirements of the client. Since the report has uncovered the structural, financial and non-financial, and functional requirements needed to develop the B2B mobile app, our client is well-informed to be able to make a sound decision as to whether to rent or buy the application, develop it himself, or even abandon the entire idea of the project. However, in the end, the ability to marshal the requisite financial and human resources; and deal with the identified potential risks that might arise during and after the project would influence the client's decision.

\section{REFERENCES}

Ahmed, S.Y. (2017). A Review of Risk Identification Approaches in the Telecommunication Domain. https://www.researchgate.net/publication/314392917_A_Review_of_Risk_Iden. tification_Approaches_in_the_Telecommunication_Domain. (Accessed on $05 \quad$ October 2021).

Aldhaban, F. (2012). Exploring the adoption of Smartphone technology: Literature review. https://www.researchgate.net/publication/261281884_Exploring_the_adoption_of_Smartphone_technology_Literature_review

Al-Naeem, F. A., Rabhi, B. B., \& Pradeep, K. R. (2005). Systematic Approaches for Designing B2B Applications. International Journal of Electronic Commerce, 9(2), 41-70.

Apollo, B. (2018). In Complex B2B Sales, Stakeholders Have More Than One Dimension. https://www.business2community.com/b2b-marketing/in-complex-b2b-salesstakeholders-have-more-than-one-dimension02111011.

Aston, B. (2016). Kickoff Meeting: The Complete Guide to Starting Projects Right. https://thedigitalprojectmanager.com/projectkickoff-meeting/.

Barnshaw, J. (2016). Facilitating Institutional Improvement through Enhanced Benchmarking. Academe, 102(2), 4-8.

Bedell, C. (2013). Mobile apps development: Defining requirements is a whole new ballgame. https://searchsoftwarequality.techtarget.com/feature/Mobile-apps-development-Defining-requirements-is-a-whole-newballgame.

Bharadwaj, A., El Sawy, O. A., Pavlou, P. A., \& Venkatraman, N. (2013). Digital Business Strategy: Toward a Next Generation of Insights. MIS Quarterly, 37(2), 471-482.

Boehm, B. W. (1991). Software Risk Management: Principles and Practices. IEEE Software, 8(1), 426-435.

Bottlik, G. P. (2017). An Introduction to the Mathematics of Planning and Scheduling, (1 $1^{\text {st }}$ ed.) Routledge; Taylor and Francis.

Chang, A. Y-P. (2016). Effects of Mobile Advertisement and Advertisement Involvement on Purchase Intention in Tourism Industry. Filomat, 30 (15), 4235-4242.

Chibesakunda, M. (2015). Reflective essay for strategic management of projects. Reflections on the BIS Project. https://www.academia.edu/21041900/REFLECTIVE_ESSAY_FOR_STRATEG

IC_MANAGEMENT_OF_PROJECTS_Reflections_on_the_BIS_Project_Stude nt_in_Work_Role.

Chinampton (2011). Application Development Plans and Gantt chart. http://blog. soton.ac.uk/chinampton/2011/02/14/applicationdevelopment-plan-and-gantt-chart/.

Chitkara, K. K. (2014), Construction project management: planning, scheduling and controlling, ( $3^{\text {rd }}$ ed.), McGraw-Hill Education.

Dahlstrom, E., Brooks, D.C., Grajek, S. \& Reeves, J. (2015). ECAR Study of Students and Information Technology. ECAR, Louisville, CO, p. 47.

Dan. S. (2017). 7 Reasons Why Small Businesses Need Mobile Apps. http://app.foundation/7-reasons-why-small-businesses-needmobile-apps/.

Daniel A. (2016). 5 Effective Uses of Mobile Technology in the Classroom. https://elearningindustry.com/5-uses-mobile-technologyin-the-classroom.

DATAREPORTAL. (July 2021). DIGITAL AROUND THE WORLD. https://datareportal.com/global-digital-overview.

Dickson, G. (1989). Corporate Risk Management. London: Witherby.

Dolly, B. \& Khanum, M. A. (2016). Requirement Elicitation in Mobile Apps: A Review, http://ijcsit.com/docs/aceit-conference2016/aceit201652.pdf.

Duffie, D., Dworczak, P. \& Zhu, H. (2017). Benchmarks in Search Markets. The Journal of Finance, 72(5), 1983-2044.

Douglass, B. L. (2016). Fundamentals of Life-Cycle Cost Analysis Article in Transportation Research. Record Journal of the Transportation Research Board.

Eversole, R. (2018). Stakeholder Analysis. https://www.researchgate.net/publication/327453706_Stakeholder_Analysis.

Fahad, I. (2016). Systematic mapping study on integration of B2B customers in ERP. (Master Thesis: Lappeenranta University of Technology. School of Business and Management). DOI: 10.13140/RG.2.2.23561.24166.

Fuller S. (2016). Life-Cycle Cost Analysis (LCCA). https://www.wbdg.org/resources/life-cycle-cost-analy sis-lcca.

Fuller, J. A., \& Robinson, M. C. (2015). Fluidized Bed Industry Benchmarking: A Five-Year Review. The Journal of Energy and Development, 41(1/2), 119-133.

Gill, M., Sridhar, S., \& Grewal, R. (2017). Return on Engagement Initiatives: A Study of a Business-to-Business Mobile App. Journal of Marketing, 81(4), 45-66.

Gotterbarn, D. \& Rogerson, S. (2005). Responsible Risk Analysis for Software Development: Creating the Software Development Impact Statement. Communications of AIS, 15(40).

Gunda S. G. (2008), Requirements Engeneering: Elicitation techniques, http://www.divaportal.org/smash/get/diva2:215169/fulltext01.

Gupta, P. D., Guha, S. \& Krishnaswami, S. S. (2013). Firm Growth and Its Determinants. Journal of Innovation and Entrepreneurship, 2(15), 1-23. 
Hartney J. (2017). Types of Project Resources. http://www.projectengineer.net/types-of-project-resources/.

Haugan, G. T. (2001). Project Planning and Scheduling. Berrett-Koehler Publishers.

Haugestad, R. (2015). Innovation Strategy for B2B Mobile Apps: a Value-centric Approach (Publication No. 3.532Mb) [Master Thesis, Norwegian University of Life Sciences]. https://brage.bibsys.no/xmlui/bitstream/handle/11250/295632/haugestad_mast er2015.pdf?sequence $=4$.

Haughey, D. (2015). Project planning a step-by-step guide. https://www.projectsmart.co.uk/project-planning-step-by-step.php.

Henry, J. (2004), Software Project Management, A Real-World Guide to Success. Boston, MA: Addison Wesley.

Hood, J. \& Nawaz, M. S. (2004). Political Risk Exposure and Management in Multi-National Companies: Is There a Role for the Corporate Risk Manager? Palgrave Macmillan Journals, 6(1), 7-18.

Jennifer B. (2017). Resource Planning for Projects: A Guide. https://www.projectmanager.com/training/resource-planning-forprojects-a-guide.

Jeon, W., Rhee, K. \& Won, D. (2012). Security Requirements of a Mobile Device Management System, https://www.researchgate.net/publication/267227402_Security_Requirements_of_a_Mobile_Device_Management_System.

Kapil, M. (2017). The undeniable importance of mobile applications and its global impact. International Journal of Advanced Science and Research ISSN: 2455-4227, 2(3), 32-36.

Kasozi, A. B. K. (2016). Designing the Benchmarks for Universities. In The National Council for Higher Education and the Growth of the University Sub-sector in Uganda, 2002-2012 (pp. 35-42). CODESRIA.

Krikor, M. (2010). IT Project Environment Factors Affecting Requirements Analysis in Service Provisioning for the Greek Banking Sector. Journal of Software Enginee ring \& Applications, 3, 858-868. requirements development:

$F A Q$. https://searchsoftwarequality.techtarget.com/feature/Softwarerequirements-development-FAQ.

Liz, C. (2018). Project management: time estimates and planning. https://www.projectsmart.co.uk/project-management-timeestimates-and-planning.php.

Michael, D. (2017). BEST PRACTICES: A New Benchmark for Ethical Compliance. GPSolo, 34(4), 64-67.

Mobile GSMA, (2018). The Mobile Economy. www.gsmaintelligence.com.

Najadat H., Alsmadi I. \& Shboul Y. (2012). Predicting Software Projects Cost Estimation Based on Mining Historical Data. ISRN Software Engineering. http://dx.doi.org/10.5402/2012/823437.

OECD (2004). ICT, E-Business and Small and Medium Enterprises. OECD Digital Economy Papers, No. 86, OECD Publishing, Paris. http://dx.doi.org/10.1787/232556551425.

Oinas-Kukkonen, H. \& Kurkela, V. (2003). Developing Successful Mobile Applications. International Conference on Computer Science and Technology, Cancun, Mexico, 50-4,

Okello-Obura, C. \& Ssekitto, F. (2015), Web 2.0 Technologies Application in Teaching and Learning. Makerere University Academic Staff

Pang, M.-S., Suh, W., Kim, J., \& Lee, H. (2009). A Benchmarking-Based Requirement Analysis Methodology for Improving Web Sites. International Journal of Electronic Commerce, 13(3), 119-162.

Passenheim, O. (2009). Project Management. Copenhagen: Bookboon.

Pizzutillo, P. (2015). Software Benchmarks and Benchmarking. https://www.castsoftware.com/blog/software-benchmarks-andbenchmarking.

Prasad, K. V. M. \& Kishore, J. K. (2013). Security Requirements Analysis For The Development Of Secure GEOSCHEMACS. International Journal of Engineering Research \& Technology (IJERT), 2(8), 2278-0181.

Project Management Institute (2013), A Guide to the Project Management Body of Knowledge (PMBOK Guide). Newtown Square, Pa.: Project Management Institute.

Rafiq, U., Bajwa, S. S., Wang, X. \& Lunesu, I. (2017), Requirements Elicitation Techniques Applied in Software Startups. 201743 rd Euromicro Conference on Software Engineering and Advanced Applications (SEAA), 141-144. doi: 10.1109/SEAA.2017.73. http://ieeexplore.ieee.org/stamp/stamp.jsp?tp=\&arnumber=8051340\&isnumber $=8051309$.

Rawassizadeh, R. (2017). Mobile Application Benchmarking Based on the Resource Usage Monitoring. https://pdfs.semanticscholar.org/4204/29735a983126ac0e4165edbba75f11ed e578.pdf.

Robertson, K. W. (2017). Benchmarking the Current Employment Statistics survey: perspectives on current research. Monthly Labor Review, 1-21,

Robertson, $\quad$ T. (2005). A useful design tool mBusiness. https://www.researchgate.net/publication/4167674_Use scenarios_A useful_design tool_for_mBusiness.

Rolstadås, A., Pinto, J. K., Falster, P. \& Venkataraman, R. (2015). Project decision chain. Project Management Journal, 46(4), 6-19.

Rose, E. (2017). The Anatomy of an Effective B2B Mobile App. https://www digitaldoughnut.com/articles $/ 2017 / \mathrm{march} / \mathrm{the}-\mathrm{anatomy-}$ of-an-effective-b2b-mobile-app.

Ryan, M. J. (2014). The role of stakeholders in requirements elicitation. INCOSE Int Symp, 24(1), 16-26.

Samsung for Business, (Aug 19, 2021). Your phone is now more powerful than your PC. https://insights.samsung.com/2021/08/19/your-phone-is-now-more-powerful-than-your-pc-3/.

Sandy, M., Aven, T., \& Ford, D. (2005). On Integrating Risk Perspectives in Project Management. Risk Management, 7(4), 7-21.

Schwalbe, C. (2004). Information Technology Project Management, $3^{\text {rd }}$ ed. Boston, MA: Thomson Publishing.

Sharp, H., Finkelstein, A. \& Galal, G. (1999). Stakeholder Identification in the Requirements Engineering Process. http://discovery.ucl.ac.uk/744/1/1.7_stake.pdf.

Smriti, J. \& Maya, I. (2011). Software Security Requirements Gathering. Instrument. International Journal of Advanced Computer Science and Applications (IJACSA), 2(7).

Strang, D. (2010). Benchmarking as a Management Technique. In Learning by Example: Imitation and Innovation at a Global Bank, (27-54). Princeton University Press.

Tira, D. (2018). What Is the Importance of Project Scheduling \& Its Role in Business Projects? https://yourbusiness.azcentral.com/importance-project-scheduling-its-rolebusiness-projects-9503.html.

Tripathi, R \& Rai, P. K. (2016). Comparative Study of Software Cost Estimation Techniques. International Journal of Advanced Research in Computer Science and Software Engineering, 6(1), ISSN: 2277 128X.

Valdellon, L. (November 1, 2020). What Are the Different Types of Mobile Apps? And How Do You Choose? https://clevertap.com/blog/types-of-mobile-apps/.

Verdon, D. (2004). Risk Analysis in Software Design. The IEEE Computer Society.

Veronica, S. M., Suhok, Silvania, H. \& Criswanto, D. N. (2014). Requirement analysis method of ecommerce websites development for small-medium enterprises, Case Study: Indonesia, International Journal of Software Engineering \& Applications (IJSEA), $5(2)$.

Vorhies, D. W. \& Morgan, N. A. (2005). Benchmarking Marketing Capabilities for Sustainable Competitive Advantage. Journal of Marketing, 69(1), 80-94.

Walid, H. (2017). 5 of the Biggest Information Technology Failures and Scares. https://www.exoplatform.com/blog/2017/08/01/5of-the-biggest-informationtechnology-failures-and-scares.

Watts, A. (2014). Project Management. Victoria, B. C.: B Ccampus. 
European Journal of Humanities and Social Sciences www.ej-social.org

World Economic Forum. (2003). Global IT Report 2002-2003. Edited by Soumitra Dutta of INSEAD, Bruno Lanvin of infoDev (World Bank) and Fiona Pauainsead of the World Economic Forum. Oxford University Press.

Mr Abdallah I. Haruna received his B.A. in Philosophy and M.Phil in African Studies from the University of Ghana. He also studied for the Master of Business Administration (MBA) degree at the University of South Wales, UK. He is currently a PhD candidate in International Relations at the Social Sciences University of Ankara, Turkey. Mr Abdallah is also an adjunct lecturer in African Studies at the Turkey-Africa Foundation. His research focuses on the influence of non-state actors on foreign policy in Ghana, Migration, Civil Society Activism in Africa, and Management Ethics. 